\title{
A mixed reality application for sketching in prototyping workshops
}

\author{
Katia Cirillo \\ Daimler AG \\ Mercedesstrasse 137 \\ 70327 Stuttgart, Germany \\ Email: katia.cirillo@daimler.com
}

\author{
Sascha Herr, Nico Koprowski, Omar Sanchez \\ Daimler AG \\ Mercedesstrasse 137 \\ 70327 Stuttgart, Germany \\ Email: sascha.herr@daimler.com \\ nico.koprowski@daimler.com \\ omar.w.sanchez@daimler.com
}

\begin{abstract}
Sketching is a method used in user-centred design to visualize first drafts of a product. In corporate environments, sketching is often employed in ideation workshops with participants of various disciplines including end users. The aim of sketching is to promote communication and create a better understanding between stakeholders. However, participants are sometimes reluctant to engage in the activity for fear of inferior drawing skills. In order to counteract this phenomenon, we designed a mixed reality application that supports users in sketching, particularly in workshop settings. Two independent user studies showed conflicting results depending on the assumed perspective. First hand users find that the application effectively supports them in creating high quality sketches with high enjoyment in the process, although they do not see creativity enhancements or higher time efficiency. In contrast, third parties that rate the produced artifacts could not distinguish between application supported and free hand sketches in terms of uniformity, comprehensibility and quality.
\end{abstract}

\section{INTRODUCTION}

$\mathbf{P}$ ROTOTYPING is a method for early evaluation of product designs in the user-centred design process. In prototyping workshops, hand-painted drawings called sketches serve to visualize initial design ideas quickly and easily. This promotes communication between participants which iteratively contributes to a better result [1]. Sketches vary according to the draftsman's knowledge and skills. This happens, for example, because users do not recognize sketched elements, such as buttons or icons.

Our contribution is to support users in sketching and to improve the quality of sketches. We aim to improve understanding of sketches. In this context, we evaluate if a mixed reality application for the Microsoft HoloLens meets the requirements as a supporting application for sketching in prototyping workshops.

\section{A. User-centred design process}

User-centred design is an iterative process of defining and specifying user requirements [2]. A prototype serves as a "representation of all or part of an interactive system, that, although limited in some way, can be used for analysis, design and evaluation" [2]. In the creation of products, multidisciplinary teams support creativity and create better solutions.

This work was supported by Daimler AG
Workshops thus provide a suitable framework for the creation of prototypes. Buxton defines sketching as the process of quickly creating handmade drawings to visualize first drafts [1]. According to the author, sketches mainly serve as means of communicating design ideas. They can be distributed to potential users for feedback. Criticism and suggestions for improvement are most valuable in early stages of development. Sketches also serve as documentation for the design process in order to keep early design decisions comprehensible later on. In sum, sketching promotes communication in design creation, enables experimentation and supports the creative process.

\section{B. Requirements analysis}

We determine the context of use on participants of prototyping workshops. One target group we address consists of persons that are well versed in the development of digital applications but cannot draw well. The other target group consists of end users of a planned product who have no experience in sketching user interfaces.

Based on the context of use, we establish the following hardware requirements for a tool that supports sketching:

1) Displaying drawing templates: The hardware should provide a possibility of displaying drawing templates that users can trace. Templates help the target group to draw professionally looking sketches and support them in sketching.

2) Use of pen and paper: In the design process, designers prefer the use of pen and paper rather than computer devices [3], [4]. That's why we determine that users do not have to sketch on computer devices. This implicates that users need their hands free for holding a pen and interact with physical objects.

3) Fast and easy set-up: As workshops take place in different premises, we set a mobile solution that includes a fast and easy set-up as a further requirement.

We determine further requirements for the software. These are derived from the definition we gave about sketching in section I-A.

4) Improvement of the quality of sketches: We aim to improve sketches by offering support to users for the drawing process. We try to establish conventions for elements in sketches. Consequently, sketches will look consistent no matter 
which user has drawn it. By this, we try to achieve a better understanding of the sketches.

5) Not limiting creativity or discussion: Our solution supports sketching and should not interfere with the goals of the method. By definition, sketching aims to support creativity. Furthermore, sketches motivate workshop participants to communicate and discuss. A requirement that we define is thus not to limit creativity or discussion.

6) Joy of use: Positive feelings while using a product supports creativity [5]. Thus we aim to develop a solution that users like to use.

\section{Related Work}

Studies that support paper prototyping with overhead projector and camera have been rated positively by participants and experts [6], [7]. Such a hardware setup is time-consuming. A study that solves this problem concerns the use of mobile devices [8]. In this approach, the drawing is not done on paper but on the devices. However, it has already been proven that in early stages of design creation, designers prefer the use of pen and paper rather than computer devices [3], [4].

Another study supports drawing with pen and paper by applying the onion skinning technique [9]. The draftsman holds a mobile device with one hand and draws underneath the device with the other hand. According to the authors, feedback was mostly positive but users considered the device too heavy and discomfortable to hold. For further work, the authors propose a solution for see-through displays.

SketchAR [10] is an application with drawing templates for Augmented-Reality. The device projects drawing templates onto a surface in the room. Users then trace the projected lines with a pen. The application includes drawing templates of everyday objects, such as animals, plants, and humans. However, elements that are necessary for use in the context of sketching digital products are missing.

An application that meets the requirements that we defined in section I-B has not been found during research. However, in the presented work, users accept applications supporting prototyping positively.

\section{Sketching Tool}

Microsofts' HoloLens [11] meets the hardware requirements of a supporting tool for sketching defined in section I-B. The head-mounted device makes it possible to project digital content onto a surface with fixed world anchors. The advantage over smartphones is that the device is head-worn, leaving hands free for drawing. There is no need of further technical equipment and cables. This is suitable for workshops, as these are often held in external premises where technical equipment varies. As hardware requirements are met with the HoloLens, the following section examines how to meet software requirements defined in section I-B.

\section{A. Concept}

The tool offers graphics of UI elements for sketching, which a user may then trace. For a better overview of the available
UI widgets, they are classified into categories. Users access the sketching mode by choosing an UI widget from the menu. A graphical visualization appears in front of a whiteboard. As support in sketching, we employ the onion skinning technique as it has been positively received in previous studies. Users step in front of the whiteboard and trace the lines of the projected element with a pen. Users can re-position, scale or rotate the element. If users have painted the element, they remove the digital projection. The hand-painted element remains on the whiteboard.

User interact with the tool by hand gestures. Audio output and speech commands are not used. We assume that audio output and speech commands distract users and create distance between user and workshop participants.

\section{B. HoloLens prototype}

The implemented prototype is shown in Fig. 1. We captured the prototype in the Unity Editor. The first image shows the sketch elements menu. The second image includes an UI widget in sketch mode and the main menu below the element. The third image displays the bounding box that allows users to scale and rotate the sketch element.

\section{USER STUDY I: USER TESTS WITH THE PROTOTYPE}

We evaluated the suitability of the HoloLens prototype in sketching workshops. We asked users to create sketches with and without the application. Afterwards, they gave feedback and rated their experiences in a questionnaire.

\section{A. Participants}

We selected test users without extensive experiences with sketching to prevent influence through routine. The sample represented users that are insecure with the sketching process. We carried out user tests with five female and four male participants. The age range was between 21 and 33 years. The mean values were 4.89 for the previous experience with smartphone apps, 1.89 for head-mounted displays and 2.89 for sketching.

\section{B. Design}

In order to evaluate the effect of the application, we conducted a within subjects design. Each test person performed one task per condition: once only with pen and paper and once with the aid of the HoloLens application.

In each condition the task for the participants was to sketch an application that was defined beforehand. It was assumed that participants were familiar with smartphone apps. We selected apps based on a survey on the use of smartphone functions [12]: chat, music player, picture gallery, news articles, and public transport. We also added a task planner app. A textual specification of the use cases ensured that participants concentrated on creating sketches instead of spending effort to understand the task. We aimed to define an environment similar to workshop situations and offered test users to address the moderator for questions regarding the content of the use case. 

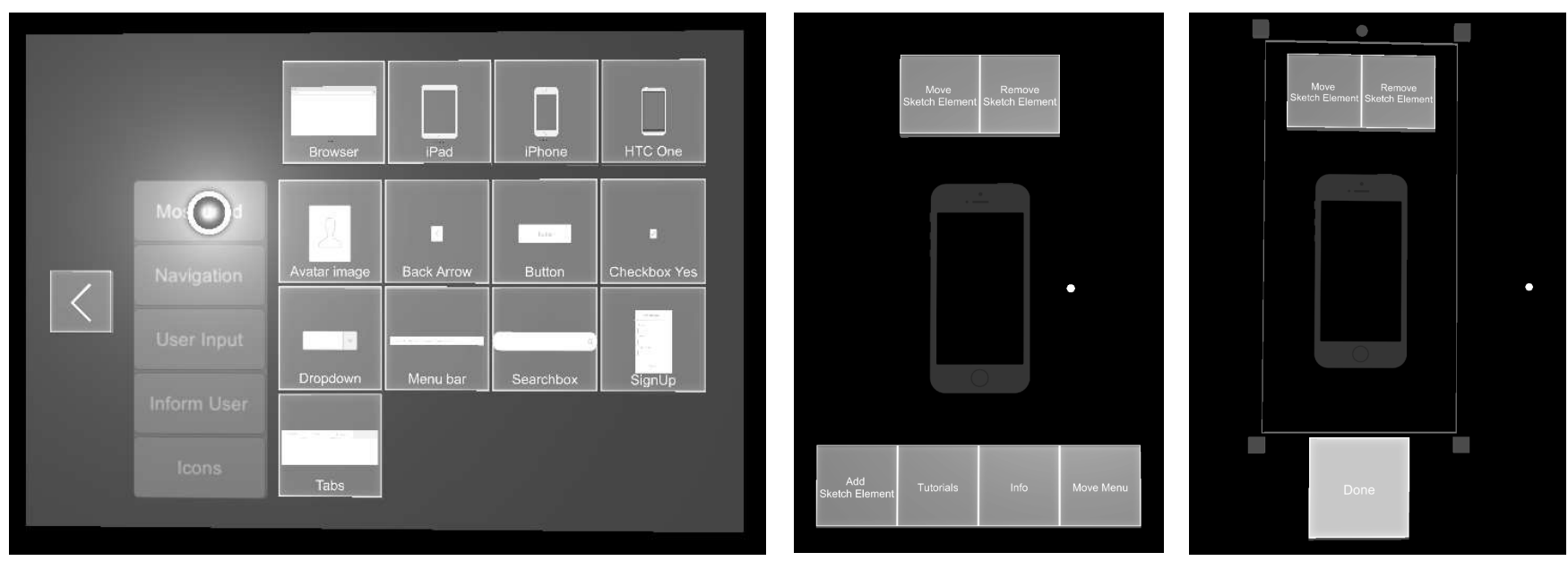

Fig. 1. Images of the HoloLens prototype from the Unity Editor. The first image (from left to right) shows the sketch elements menu, the second image shows a UI widget in sketch mode and the main menu. The third image displays the bounding box that allows users to scale the sketch element.

For each participant, we randomized the use cases and the order of conditions to avoid carry over effects. Furthermore, an equal distribution of the factors gender and prior experience was considered.

\section{Questionnaire criteria}

Participants rate criteria on a questionnaire after having performed the two tasks. The criteria included requirements from section I-B and were derived from similar work [7], [8]. In the questionnaire, users rate their extent of agreement on a Likert scale. The criteria were as follows.

1) Effectiveness: In order to answer whether the developed application is suitable for creating sketches, the effectiveness is evaluated. It is defined that the application is effective if the content needed to achieve the task is available.

2) Creativity: By definition, the method sketching supports the creative process [1]. Therefore, we evaluate whether the test users think that the application supports them in creativity.

3) Time-efficiency: Experts determine rapid execution as an important property of prototyping tools [7]. Since time of execution is an uncontrollable constant that varies between people, it is not feasible to measure it. Instead, we query an assessment of the test users.

4) Enjoyment: Positive feelings support creativity [5]. An expert also classifies enjoyment as important for prototyping tools [7]. We ask users whether the solution of the task was more fun with the support of the application.

5) Quality of results: We assume that the application is more likely to be used if users consider the results to be more professional than without the application.

\section{Procedure}

We carry out the user tests with each test person individually. After an introduction, test subjects read through the first task and start with the head-worn HoloLens. All test users operate the HoloLens by hand. Test users solve the tasks uprightly in order to simulate the workshop scenario.

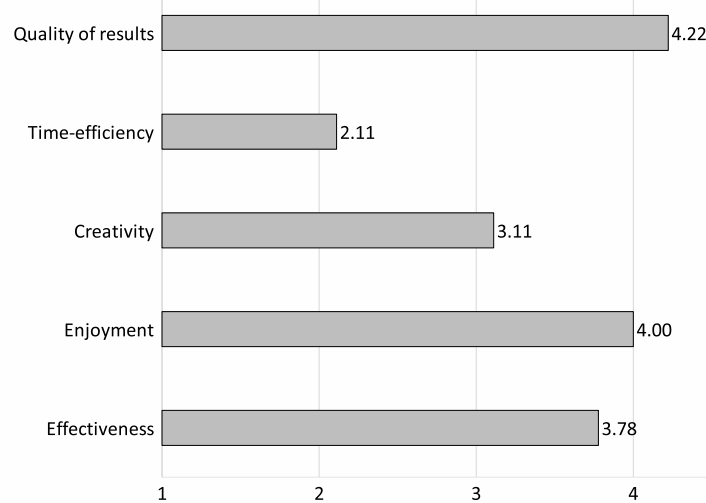

Fig. 2. Mean values of the criteria quality of results, time-efficiency, creativity, enjoyment, and effectiveness. The scale ranges from one (This does not apply) to five (This applies)

They start to sketch with a ballpoint pen on paper sheets. After completing the first task, the test users read and solve the second task. The moderator removes the sheets of the first round so that the user can no longer look at them. The test users fill out the questionnaire after completing both assignments.

\section{E. Results}

The questionnaire evaluates the criteria quality of results, time-efficiency, creativity, enjoyment, and effectiveness. The results are shown in Fig. 2. The graphic includes the mean value of the ratings from the test users per criterion.

The mean value for the quality of results represents the highest value $(\mathrm{M}=4.22, \mathrm{SD}=0.83)$. The test users agreed that the solution of the task was more fun with the system $(\mathrm{M}=4.00$, $\mathrm{SD}=0.87$ ). They slightly agree that the application offered the contents they needed $(\mathrm{M}=3.78, \mathrm{SD}=0.83)$. They neither agree 
nor disagree in average that the application supports their creativity $(\mathrm{M}=3.11, \mathrm{SD}=1.36)$. The only statement that test users do not agree with is the time-efficiency of the application $(\mathrm{M}=2.11, \mathrm{SD}=1.27)$.

In a free text field for further notes, test users referred to the HoloLens application and noted more consistency in sketches; usage became easier after adaption; more enjoyment; added value of support with drawing templates; suitability for large elements instead of small elements; usage for small elements is time-consuming and impractical; no additional support of creativity; support for visualization of symbols; usage better for copying the element instead of tracing lines directly from drawing template.

\section{USER STUDY II: EVALUATION OF THE SKETCHES}

We decided to conduct a subsequent study in order to evaluate the sketches from study I. Another group of participants evaluates the sketches which were created with and without the help of the application.

\section{A. Participants}

We select participants without any specific prior knowledge for the assessment of sketches. As this work is supported by Daimler AG, we determine German employees of the company as population and select a random sample. The number of employees of Daimler AG on 31.12.2017 in Germany was 172,089 [13]. In the online survey, 70 male and 25 female persons participate. The age is given in ranges of 20-29 years (31 participants), 30-39 (34 participants), 40-49 (16 participants), 50-59 (13 participants) and 60-69 (one participant). No person is younger than 20 years or older than 69 .

\section{B. Design}

We plan the study with a within subjects design. We use the resulting sketches from the first study as stimulus. Each participant evaluates sketches with both features, i.e. with and without HoloLens application. Participants give their rating on a Likert scale in an online questionnaire.

Each participant evaluates three out of six use cases of each stimulus. To ensure that the same prerequisites apply to both stimuli, the tool stores each use case once per stimulus. We randomize the selected use cases and order of their occurrence. We give no information about the context in which the sketches were created. Users evaluate each use case independently of the other.

\section{Questionnaire criteria}

With the criteria we selected, we aim to evaluate if the application supported sketches look better.

1) Comprehensibility: Sketches are used to communicate design ideas in an understandable way [1]. By this criterion we determine whether participants recognize concepts and elements, such as buttons, arrows or icons.

2) Basis of discussion: Sketches serve as a basis of discussion and means of getting user feedback [1]. The survey participants evaluate if the sketches serve as a basis of discussion.
3) Uniformity: We assume that more uniformity in the presentation of the sketches leads to a better understanding. We define uniformity in sketches if users have chosen the same representation of elements.

4) Quality of results: The representation of sketches varies depending on the draftsmans' skills or the degree of maturity of the sketches. To counteract this effect, the application aims to improve it. Participants evaluate whether the sketches appear to be made in a professional context, for example by an experienced team or with the help of a tool.

\section{Procedure}

Participants evaluate the sketches in an online survey. Each questionnaire starts with an introduction to sketching and the evaluation criteria. Each of the following six pages shows a use case consisting of three sketches and a Likert scale.

\section{E. Results}

For the results we only consider questionnaires where the participant reached the last page. Table I shows mean values and standard deviations for the evaluated criteria.

TABLE I

Mean Values and STANDARD DEVIATIONS FOR THE EVAluated CRITERIA IN USER STUDY II.

\begin{tabular}{l|ll|ll}
\multirow{2}{*}{ Criterion } & \multicolumn{2}{|c|}{ Conventional sketches } & \multicolumn{2}{|c}{ HoloLens sketches } \\
& Mean & SD & Mean & SD \\
\hline Uniformity & 3.14 & 1.35 & 3.18 & 1.35 \\
Comprehensibility & 3.25 & 1.7 & 3.06 & 1.47 \\
Basis of discussion & 3.26 & 1.74 & 3.19 & 1.37 \\
Quality of results & 2.36 & 1.11 & 2.66 & 1.34
\end{tabular}

\section{DISCUSSION}

The suitability of the application for sketching is derived from the criteria time-efficiency and effectiveness, which were evaluated in the user tests. The results show that sketching takes longer. Users needed time to familiarize themselves with the device and the application. Test users searched for suitable elements. Additionally, they needed more time to draw elements. Interactions are unfamiliar with first-time users. However, these limitations can be improved through training. Not all test users stated that the required content was provided. This can be improved easily by adding additional elements.

Based on user study II, we do not see any clear effects in the application supported sketches. We could not prove that sketches get more uniform with our tool. It follows that we cannot assess whether more uniformity in the presentation of sketches has a positive effect on comprehensibility.

Nevertheless, we recognize that test users were positive about our application. Test users believe that they have achieved more professional results. In addition, they enjoy solving the task with the application. We assume that an improvement in hardware and the application will lead to better results in time-efficiency.

In summary, the evaluation revealed that test users rate the application positively in terms of quality of results and enjoyment. Accordingly, the added value of using HoloLens 
for sketching lies more in the use of the application than in resulting sketches. The application seems suitable to solve inhibitions of participants of prototyping workshops towards the method sketching. Thus, the application appears to be useful to familiarize participants with sketching. As test users say they have had more fun and think that the resulting sketches are more professional, it is conceivable to involve participants of workshops in the sketching process. Through the increased involvement of participants, we could encourage communication and discussion. Since this is one of the main reasons to use sketching, we suggest further investigation about the benefits of the application.

\section{CONCLUSION AND Future WORK}

We investigated how a mixed reality application supports the method sketching in prototyping workshops. We described the suitability of the Microsoft HoloLens as a device for an application in sketching. The target group for the application are participants of prototyping workshops. We proposed drawing templates to support the sketching process and familiarize participants with the method.

We implemented a prototype on HoloLens. The device projects drawing templates onto a wall where users then trace the element with pen on a paper. In user tests, we evaluated the added value of the tool. As a result, the application has been well received by users. We therefore propose to carry out further studies. It seems interesting to put the focus of evaluation on user experience to determine the involvement of participants in the sketching process.

For better results in an evaluation of usability and user experience, we suggest to continue development of the prototype by expanding the range of UI widgets and improving the interaction concept. We suggest minimizing direct interaction by the user within the application.

Another relevant criterion is technology acceptance. If users do not want to use the device in workshops because they feel restricted or not taken seriously, the application will not be used. In addition, we suggest the evaluation of the suitability in collaborative group work. The application can furthermore be extended so that several HoloLens devices can be connected so that users can work together in remote work scenarios.

\section{REFERENCES}

[1] W. Buxton, Sketching user experiences : getting the design right and the right design. Elsevier/Morgan Kaufmann, 2007. ISBN 0123740371

[2] International Organization for Standardization, "Ergonomics of humansystem interaction - Part 210: Human-centred design for interactive systems," 2010. [Online]. Available: https://www.iso.org/obp/ui/ $\{\backslash \#\}$ iso: std:52075:en

[3] D. J. Cook and B. P. Bailey, "Designers' use of paper and the impliations for informal tools," Ozchi, pp. 1-10, 2005.

[4] M. W. Newman and J. A. Landay, "Sitemaps, storyboards, and specifications," in Proceedings of the conference on Designing interactive systems processes, practices, methods, and techniques - DIS '00. New York, New York, USA: ACM Press, 2000. doi: 10.1145/347642.347758. ISBN 1581132190 pp. 263-274.

[5] A. Filipowicz, "From positive affect to creativity: The surprising role of surprise," Creativity Research Journal, vol. 18, no. 2, pp. 141-152, 2006. doi: $10.1207 / \mathrm{s} 15326934 \mathrm{crj} 1802 \_2$

[6] J. Laviole and M. Hachet, "PapARt: Interactive 3D graphics and multitouch augmented paper for artistic creation," IEEE Symposium on $3 D$ User Interfaces 2012, 3DUI 2012 - Proceedings, pp. 3-6, 2012. doi: 10.1109/3DUI.2012.6184167

[7] B. Bähr, Prototyping of user interfaces for mobile applications, ser. TLabs Series in Telecommunication Services. Cham: Springer International Publishing, 2017. ISBN 978-3-319-53209-7

[8] Q. Su, W. H. A. Li, J. Wang, and H. Fu, "EZ-sketching: Threelevel optimization for error-tolerant image tracing," ACM Trans. Graph., vol. 33, no. 4, pp. 54:1-54:9, 2014. doi: 10.1145/2601097.2601202

[9] C. Lins, E. Arruda, E. Neto, R. Roberto, V. Teichrieb, D. Freitas, and J. M. Teixeira, "Animar: Augmenting the reality of storyboards and animations," Proceedings - 2014 16th Symposium on Virtual and Augmented Reality, SVR 2014, pp. 106-109, 2014. doi: 10.1109/SVR.2014.40

[10] Sketchar.tech, "SketchAR." [Online]. Available: http://sketchar.tech/

[11] Microsoft, "Buy Microsoft HoloLens for Developers and Business I HoloLens." [Online]. Available: https://www.microsoft.com/de-de/ hololens/buy

[12] Statista, "Smartphone features: usage in Germany 2017 । Survey." [Online]. Available: https://www.statista.com/statistics/436515/ smartphone-features-usage-germany/

[13] Daimler AG, "Daimler Unternehmensprofil." [Online]. Available: https://www.daimler.com/dokumente/investoren/berichte/ geschaeftsberichte/daimler/daimler-ir-daimler-im-ueberblick-2017.pdf 